\title{
Chemical Agent Material Identification Code
}

National Cancer Institute

\section{Source}

National Cancer Institute. Chemical Agent Material Identification Code. NCI Thesaurus.

Code C161504.

The unique identifier code assigned by the manufacturer or distributor to a specific quantity of chemical challenge agent. This may include batch number, lot number, batch/lot number, etc. 